\title{
Delayed Eruption due to Concurrent Occurrence of Compound Odontoma and Supernumerary Tooth in the Maxillary Anterior Region: A Rare Case Report
}

\author{
${ }^{1}$ Megha Gupta, ${ }^{2}$ Suman Panda
}

\begin{abstract}
Frequently seen benign odontogenic tumors are the odontomas which consists of dental tissue. They are regularly asymptomatic but can cause troubles in the teeth eruption manifesting clinically as late eruption or deflection. They are identified due to retained primary teeth or on routine radiographic examination. Supernumerary teeth are in addition to the normal complement. These teeth usually have the normal shape. Odontomas and supernumerary teeth are categorized as separate entities. However, possibly they have the expression of the similar pathologic process, which might be due to a malformation or a hamartoma.

This paper describes an uncommon case of retained primary teeth because of the presence of compound odontoma as well as supernumerary teeth in the anterior maxillary region of a 10-year-old boy. Surgical intervention was achieved to remove the compound odontoma and the supernumerary tooth. The permanent incisors erupted favorably after one and a half years. This case also highlights the need of early finding of odontogenic anomalies that enables a less complex treatment; and better prognosis for the patient.
\end{abstract}

Keywords: Compound odontoma, Delayed eruption, Mesiodens, Retained primary teeth, Supernumerary teeth.

How to cite this article: Gupta M, Panda S. Delayed Eruption due to Concurrent Occurrence of Compound Odontoma and Supernumerary Tooth in the Maxillary Anterior Region: A Rare Case Report. J Contemp Dent 2018;8(3):144-147.

Source of support: Nil

Conflict of interest: None

\section{INTRODUCTION}

Most common odontogenic tumors of the jaws are odontomas. Their slow growth and non-malignant nature describe them. Broca in 1866 gave the term "odontoma." He defined odontoma as "a tumor of overgrowth of complete dental tissue."1 Odontomas are composed of enamel and dentin; however may have a definite amount of cementum and pulp. ${ }^{2}$ Odontomas are hamartomatous lesions and not true neoplasms since the epithelial

\footnotetext{
${ }^{1,2}$ Assistant Professor

1,2Department of Preventive Dental Sciences, Division of Pedodontics, College of Dentistry, Jazan University, Jizan, Kingdom of Saudi Arabia

Corresponding Author: Megha Gupta, Assistant Professor, Department of Preventive Dental Sciences, Division of Pedodontics, College of Dentistry, Jazan University, Jizan, Kingdom of Saudi Arabia, e-mail: meghamcods@gmail.com
}

and mesenchymal cells and tissues of an odontoma are lacking in structural arrangement; however can appear normal. ${ }^{3}$

The etiology of odontoma is still not well understood. Local traumas or infections can cause odontomas. ${ }^{4}$ Radiographically odontomas are seen as dense radiopaque lesions with clear external margins which are encircled by a thin radiolucent zone. ${ }^{5}$

The World Health Organization (WHO) in 2005, has categorized odontomas as; complex and compound odontomas.

\section{Complex odontoma}

The dental tissues are formed well but display an amorphous and disorganized arrangement.

\section{Compound odontomas}

The dental tissues are usual and organized in a systematic pattern, but their conformation and size are altered, giving rise to multiple small teeth like elements called denticles or odontoids. ${ }^{6}$

Odontomas situated in the anterior area of the maxilla are generally compound, while those located in the posterior parts especially the mandible are complex types. ${ }^{2}$

Management consists of surgical elimination of these calcified structures. The prognosis after management is favorable, with low relapse. ${ }^{4,5}$

Supernumerary teeth are those in surplus of the normal complement. ${ }^{7}$ They can occur in any area of the dental arch; though there is a high preference for the anterior maxilla. ${ }^{8}$ It can occur as single or multiple and can present as unilaterally or bilaterally. The occurrence of supernumerary teeth as single accompanying with the permanent teeth is frequently found in the anterior maxilla and is called as mesiodens. ${ }^{9}$ Numerous supernumerary teeth are usually accompanying with different syndromes.

The precise etiology of supernumerary teeth is still not known, but two theories have been proposed. According to the dichotomy concept of tooth germ," the tooth bud splits into two equal or different sized parts, which results in the formation of two teeth of equal size or one normal and one dysmorphic tooth respectively. Localized and independent hyperactivity of dental lamina may also be 


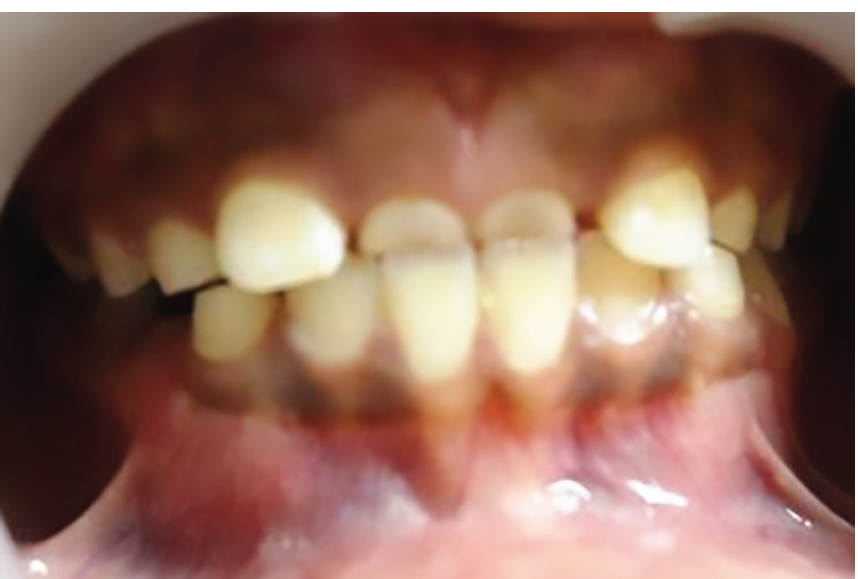

Fig. 1: Intra-oral view showing retained primary maxillary central incisor

answerable for the formation of supernumerary teeth."10 Surgical elimination is indicated in most of the cases.

Odontomas and supernumerary tooth can have a common origin. Odontomas, though classified as tumors; stop growing in volume once they are mineralized. This feature is not seen with other tumors of the body, but it does match with that of the supernumerary tooth. The site of occurrence of both; compound odontoma and supernumerary teeth is maxillary anterior region. ${ }^{11}$

\section{CASE REPORT}

A 10-year-old boy came to the Pedodontics department with the complaint of two primary maxillary teeth in the center. However, the permanent maxillary lateral incisor had erupted in the oral cavity (Fig. 1). This was a significant concern for the father, who was extremely apprehensive and concerned about the missing permanent teeth in the maxillary anterior region of the boy. The medical and family history was non-contributory. The patient was moderately built and nourished.

The panoramic radiograph showed the presence of unerupted bilateral permanent maxillary central incisors, with incomplete root formation. Also, two small mineralized radiopaque structures concerning the root of primary left central incisor and the crown of right permanent central incisor were seen. A mesiodens in relation to the crown of the left maxillary permanent central incisor was also seen (Fig. 2).

On the foundation of the clinical and radiographic findings; provisional diagnosis of supernumerary teeth, i.e., mesiodens and compound odontoma was made. A treatment plan to extract the retained primary maxillary central incisors and surgically remove the mesiodens and compound odontoma were made. The routine blood investigations were performed, and the patient was posted for surgery.

After achieving adequate local anesthesia, the retained primary maxillary central incisors were

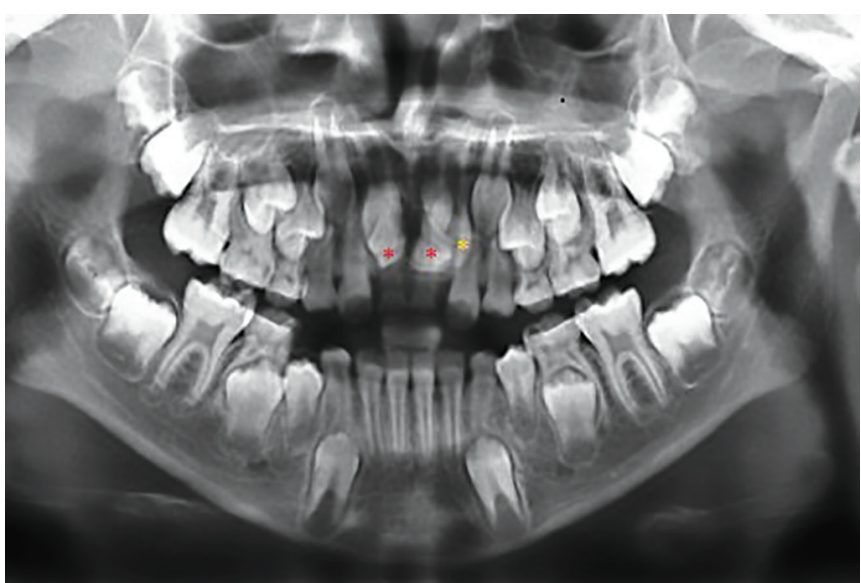

Fig 2: Panoramic view preoperative showing the mesiodens and compound odontoma in the maxillary anterior region

extracted. On the buccal and palatal side, a mucoperiosteal flap was reflected from the mesial surface of permanent right lateral incisor to the mesial surface of the permanent left central incisor. A window was made over the buccal cortical bone, using a round surgical bur under constant irrigation with saline solution and the two compound odontoma were removed (Fig. 3). The unerupted permanent maxillary central incisor could be seen. Mesiodens was present palatally and beneath the permanent central incisor of the left side. Guttering was done around the permanent central incisor of the left side, and the mesiodens was removed. So, a total of five mineralized structures (two primary central incisors, one mesiodens, and two compound odontoma) were removed, without disturbing the permanent maxillary central incisor crowns (Fig. 4).

A postoperative radiograph was taken to ensure that no calcified structures are remaining. Thereafter, the irrigation of the surgical area was done with a povidoneiodine-saline solution. After hemostasis was achieved, the flap was approximated and closed with silk sutures; which were removed after a week. Postoperative written and oral instructions related to ingestion of soft and cold meals, the maintenance of good oral hygiene, abstaining

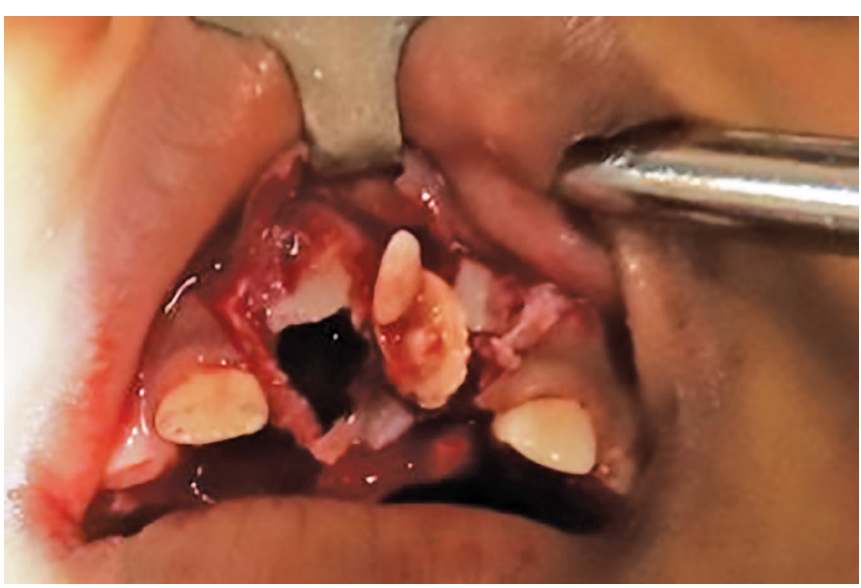

Fig. 3: Surgical removal 


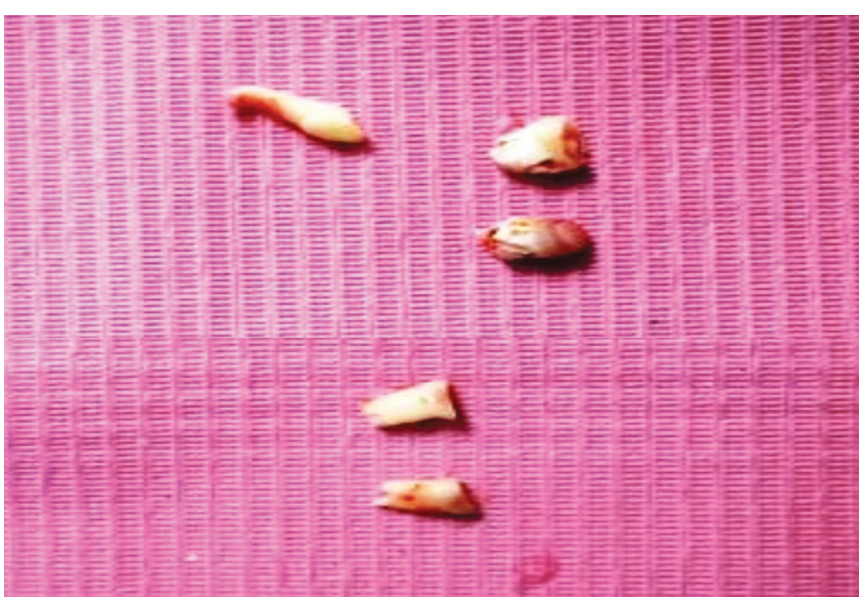

Fig. 4: The extracted mineralized structures: 2 primary central incisors, 2 compound odontoma and supernumerary mesiodens

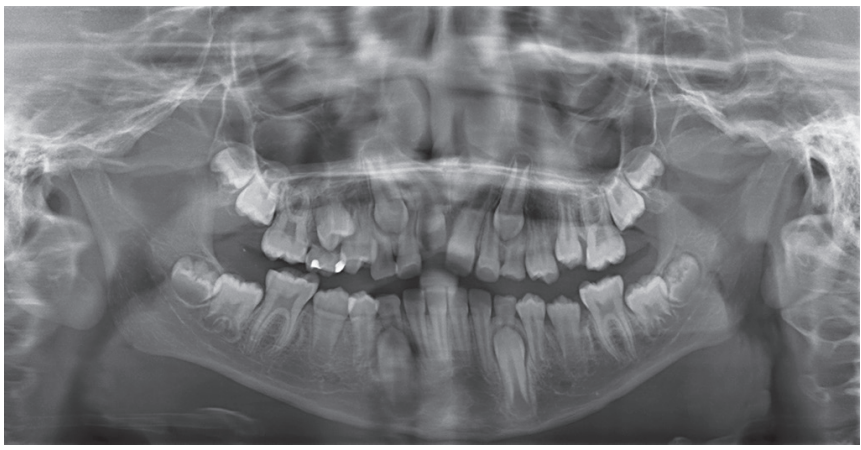

Fig. 6: Panoramic view six months post - surgery

from physical exercise through the first 48 hours and managing of pain were given to the patient's mother. Healing was uneventful.

The histopathological diagnosis confirmed the presence of a compound odontoma which showed eosinophilic dentinal tissue and an area exhibiting loose connective tissue characteristic of normal pulp (Fig 5). Follow-up radiograph after six months showed favorable eruption pattern (Fig. 6).

The permanent maxillary central incisor erupted favorably after one and a half year (Figs 7 and 8 ). The patient is under regular follow-up.

\section{DISCUSSION}

Odontomas are benign, hamartomatous developmental abnormalities of odontogenic origin. It appears as small, single or multiple radiopaque lesions on routine radiographic examination. ${ }^{12}$ Odontomas constitute $22 \%$ of all the odontogenic tumors of the jaws. ${ }^{2}$

Recent studies propose that compound odontomas and supernumerary teeth are resulting from the production of groups of epithelial cells rising from limited dental lamina hyperactivity which is connected to teratogenic or genetic stimuli. Stimuli and regulator aspects derivative from the dental lamina and/or papillae perhaps condition these cells to develop near atrophy or to dental structures.

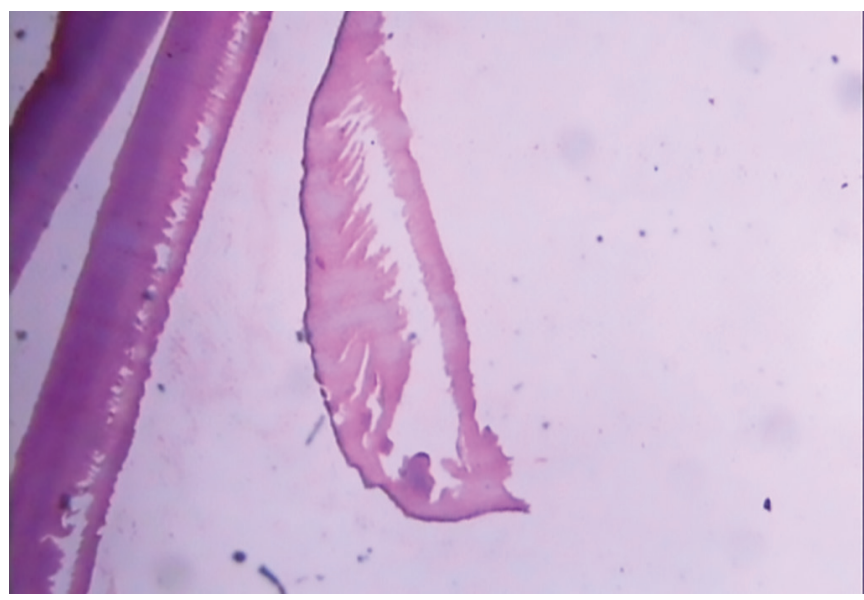

Fig. 5: Histological presentation of compound odontoma

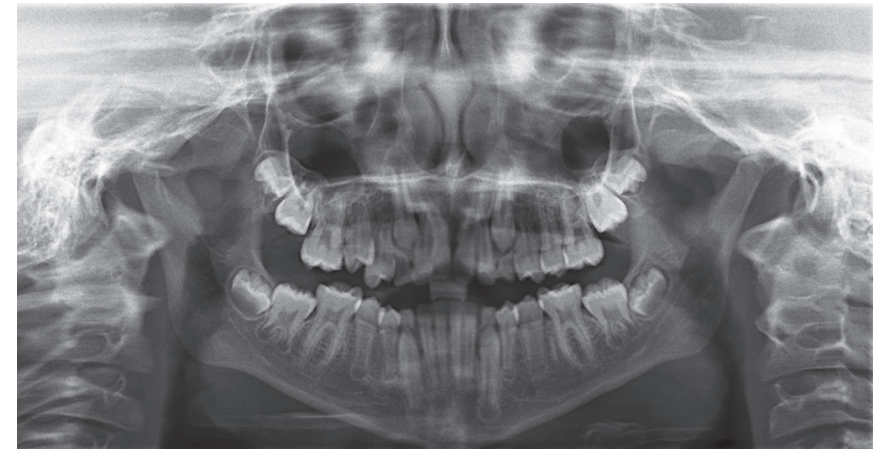

Fig. 7: Intra-oral view showing complete eruption of both the Incisors

The source of these stimuli is not entirely known while they are undoubtedly site and time related. ${ }^{11}$

Odontomas are also supplementary with various pathological situations like local trauma, inflammatory processes, mature ameloblast and variations in the genetic constituent accountable for regulatory dental growth..$^{13}$ Odontomas are also seen in syndromes like Basal cell nevus syndrome, Tangier disease, Gardner's syndrome or Hermann's syndrome. ${ }^{5}$ Common particles are articulated in the normal tooth forming as well as

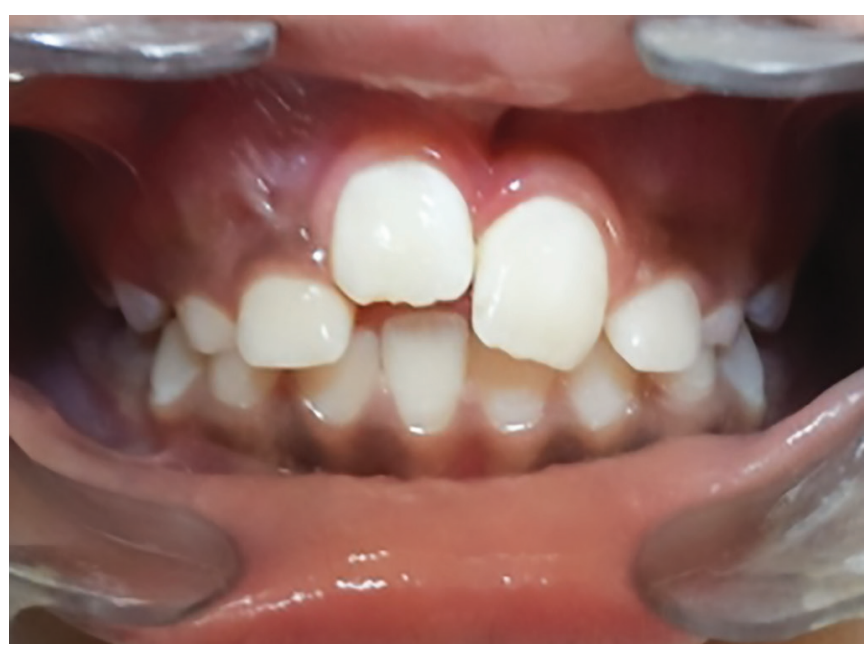

Fig. 8: Intra-oral view showing the complete eruption of both the incisors 
the odontogenic tumor cells. This could be due to recapitulation and overexpression of tumor-specific genetic programs transcribed during normal odontogenesis. ${ }^{14}$ The common of odontomas located in the anterior region of the maxilla is compound, whereas odontomas located in the posterior areas, particularly in the mandible are complex odontomas. ${ }^{13,15}$

Radiographically, odontomas seem as thick radioopaque lesions having protuberant outside margins enclosed by a thin radiolucent zone. ${ }^{5,15}$ Radiographic examination is an active method of distinguishing amongst the two types of odontoma. The compound odontoma, radiographically displays well-ordered abnormal teeth or tooth-like structure, as seen in our case while a complex odontoma looks as an irregularly shaped oval radio opacity bounded by a distinct thin radiolucent rim. ${ }^{12}$ Radiographic, as well as histological examination established the presence of a compound odontoma in our case. The concurrent incidence of supernumerary teeth (Mesiodens) and compound odontoma in our case made it a challenging one.

Odontomas are treated by conservative surgical removal; ${ }^{1,2}$ as it contains various tooth preparations that can influence to cystic change. ${ }^{16}$ A study reported that in a huge majority of cases of odontomas, the neighboring teeth undergo pathologic changes like devitalization, malformation, aplasia, malposition and delayed eruption. ${ }^{17-19}$ They can also undergo a cystic transformation. Preferably, odontomas necessarily may be removed as soon as the permanent teeth nearby to the lesion show about one half of their root progress. This will safeguard the security of the normal permanent teeth and stop interferences with their eruption. ${ }^{17-19}$ At the time of extraction, in our case, the roots of the permanent central incisor were nearly two-thirds formed, which ensued in a favorable eruption.

To conclude, both the compound odontoma and the supernumerary tooth were surgically removed, without producing any injury to the original permanent tooth germ. Since the root development of both the permanent maxillary central incisors was not complete; the teeth were left to erupt on their own. The permanent teeth erupted favorably after one and a half years. The case is exclusive, as there was a coexistence of the supernumerary tooth and two compound odontomas in the maxillary anterior region, which; to the best of our knowledge has never been reported so far.

\section{CONCLUSION}

A detailed clinical examination and radiographic investigation must be attained for all the child patients who come with clinical indication of the delayed eruption, tooth displacement or missing tooth without a history of trauma.
Early diagnosis will enable the clinician to implement a less complicated and simpler treatment and safeguards better prognosis.

\section{Ethical Clearance}

Prior, written informed consent was obtained from the patient's parents. Ethical clearance was received from the institutional ethical committee, (IRB No: 2016-02).

\section{REFERENCES}

1. Cohen DM, Bhattacharya I. Ameloblastic fibroma, ameloblastic fibro-odontoma, and odontoma. Oral Maxillofac Surg Clin North Am. 2004 Aug;16(3):375-384.

2. Amado Cuesta S, Gargallo Albiol J, Berini Aytés L, Gay Escoda C. Review of 61 cases of odontoma. Presentation of an erupted complex odontoma. Med Oral, 2003 Nov-Dec;8(5):366-373.

3. Shekar S, Rao RS, Gunasheela B, Supriya N. Erupted compound odontoma. J Oral Maxillofac Pathol. 2009 Jan;13 (1):4750.

4. Dagstan S, Goregen M, Miloglu O. Compound odontoma associated with maxillary impacted permanent central incisor tooth: a case report. Internet J Dental Sci . 2007;5:1-6

5. White SC, Pharoah MJ. Benign tumors of the jaws. In Oral Radiology: Principles and Interpretation. 5th ed. Missouri: Mosby; 2004. pp 424-428.

6. Barnes L, Eveson JW, Reichart P, Sidransky D. Eds., World Health Organization Classification of Tumours. Pathology and Genetics of E558 Head and Neck Tumours. 2005; IARC Press, Lyon, France.

7. Bhaskar SN. Synopsis of Oral Pathology. St. Louis: C.V. Mosby 1961. pp17-25.

8. Nasif MM, Ruffalo RC, Zullo T. Impacted supernumerary teeth: A survey of 50 cases. J Am Dent Assoc 1983;106:201- 204.

9. Zhu JF, Marcushamer M, King DL, Henry RJ. Supernumerary and congenitally absent teeth: A literature review. J Clin Pediatr Dent 1996; 20:87-95.

10. Liu JF. Characteristics of premaxillary supernumerary teeth: A survey of 112 cases. ASDC J Dent Child 1995; 62:262-265.

11. Pippi R. Odontomas and supernumerary teeth: is there a common origin? Int J Med Sci. 2014;12;11(12):1282-1297.

12. Waldron AC. Odontogenic cysts and tumors. In Oral and Maxillofacial Pathology, B.W. Neville. 5th ed. WB Saunders, Philadelphia, USA 2004; pp. 631-632.

13. Hitchin AD. The aetiology of the calcified composite odontomes. Br Dent J. 1971 Jun 1; 130(11):475-482.

14. Papagerakis P, Peuchmaur M, Hotton D, Ferkdadji L, Delmas $\mathrm{P}$, Sasaki $\mathrm{S}$, et al. Aberrant gene expression in epithelial cells of mixed odontogenic tumors. J Dent Res 1999;78:20-30

15. Kaban LB. Pediatric Oral and Maxillofacial Surgery. Philadelphia : Saunders, 1990, pp111-112.

16. Suri L, Gagari E, Vastardis H. Delayed tooth eruption: Pathogenesis, diagnosis, and treatment. A literature review. Am J Orthod Dentofacial Orthop 2004;126(4):432-445.

17. De Oliveria BH, Campos V, Marcal S. Compound odontomadiagnosis and treatment: three case reports. Pediatr Dent 2001;23(2):151-157.

18. Ali FM, Aisha Y, Shadia O, Ghadi D, Wejdan A, Areej A. Odontome in maxillary anterior region causing impaction of permanent central incisor tooth: A case report. International Journal of Research Studies in Medical and Health Sciences 2017;2(9):1-4.

19. Ali FM, Al-Iryani GM, Zakari AH, Hakami AO, Kinani AA. First reported case of both deciduous and permanent canine impaction due to odontome. IJSS Journal of surgery 2017;3(5):21-23. 